\title{
Muséologies
}

Les cahiers d'études supérieures

muséologies

\section{La conservation des oeuvres à composante technologique}

\section{Cindy Veilleux}

Volume 2, numéro 1, octobre 2007

URI : https://id.erudit.org/iderudit/1033599ar

DOI : https://doi.org/10.7202/1033599ar

Aller au sommaire du numéro

Éditeur(s)

Association Québécoise de Promotion des Recherches Étudiantes en Muséologie (AQPREM)

ISSN

1718-5181 (imprimé)

1929-7815 (numérique)

Découvrir la revue

Citer cet article

Veilleux, C. (2007). La conservation des oeuvres à composante technologique. Muséologies, 2(1), 86-103. https://doi.org/10.7202/1033599ar

\section{Résumé de l'article}

Pluridisciplinarité, avancées technologiques et utilisation de nouveaux médias par les artistes en art contemporain secouent les fondements même de la conservation curative et préventive. Ces changements demandent aux musées l'adoption de nouvelles politiques culturelles mieux adaptées à la réalité d'aujourd'hui. L'auteur s'interroge ainsi sur le potentiel d'une méthodologie combinant un questionnaire de conservation et un entretien avec l'artiste, vu comme un acteur déterminant au coeur du processus.
Ce document est protégé par la loi sur le droit d'auteur. L'utilisation des services d’Érudit (y compris la reproduction) est assujettie à sa politique d'utilisation que vous pouvez consulter en ligne.

https://apropos.erudit.org/fr/usagers/politique-dutilisation/ 


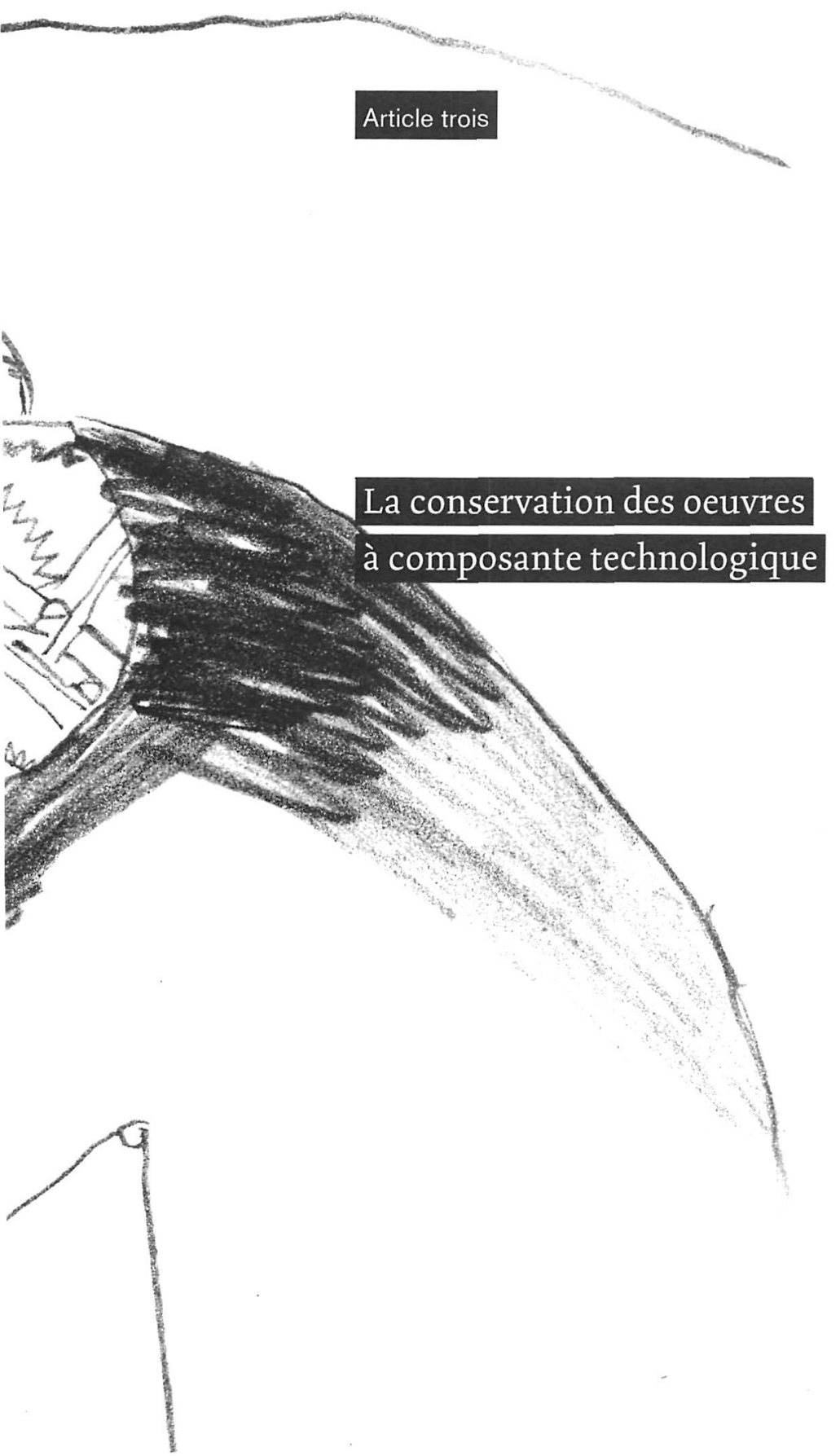


Pluridisciplinarité, avancées technologiques et utilisation de nouveaux médias par les artistes en art contemporain secouent les fondements même de la conservation curative et préventive. Ces changements demandent aux musées l'adoption de nouvelles politiques culturelles mieux adaptées à la réalité d'aujourd'hui. L'auteur s'interroge ainsi sur le potentiel d'une méthodologie combinant un questionnaire de conservation et un entretien avec l'artiste, vu comme un acteur déterminant au cœur du processus.

CINDY VEILLEUX EST TITULAIRE D'UN BACCALAURÉAT EN HISTOIRE DE L'ART ET EN ARTS VISUELS, L'AUTEURE A ÉGALEMENT COMPLÉTÉ, EN 2006, UN DIPLÓME DE DEUXIĖME CYCLE EN MUSÉOLOGIE À L'UNIVERSITÉ LAVAL, ÉTUDES TOURNÉES ESSENTIELLEMENT VERS LA CONSERVATION DU PATRIMOINE. ELLE A OCCUPÉ LE POSTE DE SPÉCIALISTE EN DOCUMENTATION DES COLLECTIONS AU SEIN DU RÉSEAU INFO-MUSE DE LA SOCIÉTÉ DES MUSÉES QUÉBÉCOIS (SMO) ET TRAVAILLE PRÉSENTEMENT AU SEIN DU MÊME ORGANISME À TITRE D'AGENTE DE RECHERCHE ET DE LIAISON POUR LE SERVICE DE LA FORMATION ET DU DÉVELOPPEMENT PROFESSIONNEL. 
[1]

SERVICE DE TECHNOLOGIE DE LINFORMATION.

BIBLIOTHĖQUE NATIONALE DU

CANADA.

La conservation de

l'information numérique : ses

divers aspects et la situation

actuelle. <http://cc.msncache.

com/cache.px?q=

$1994680634543 \&$ lang $=\mathrm{fr}-$

CA\&FORM $=$ CVRE $>$

(consulté en mars 2006).

[2]

Heureusement, de multiples projets expérimentaux et mobilisateurs investiguent et alimentent en ce moment le réseau des arts médiatiques.

Les efforts soutenus par

les initiatives de différents

organismes doivent donc profiter

d'une mise en commun, en

adoptant une sélection

rationnelle de l'information.

Exemplaire à cet égard,

la fondation Daniel Langlois

pour l'art, la science et la

technologie (FDL) abonde en

ce sens en promettant

l'unification des résultats,

de même que l'accessibilité

des outils développés par

les membres de l'équipe

multidisciplinaire qu'elle a

mise sur pied pour le projet

Documentation et conservation

du patrimoine en arts

médiatiques (DOCAM).

Pour des détails, consulter

le site Internet de DOCAM :

<http://www.docam.ca>

(consulté en mars 2006).

[3]

BUREAUD, Annick,

Nathalie LAFFORGUE

et Joël BOUTTEVILLE.

Art et technologie : la monstration. Rapport d'étude à la Délégation aux arts plastiques. Paris : Ministère de la Culture, novembre 1996, p. 138. Pour télécharger ou consulter ce document en ligne : <http:// www.olats.org/OLATS/livres/ etudes/monstration/ presentationsite.htm>.
"Les mesures de conservation ont pour but de rendre un document ou un artefact - numérique ou autre - accessible dans une forme utilisable pour longtemps. ${ }^{[11}$ " Cette citation, tirée d'un article d'Alison Bullock, portant sur la conservation de l'information numérique illustre bien le défi actuel que les professionnels de la muséologie doivent relever.

L'avancée rapide des technologies et ses percées remarquées dans les différentes sphères de la société invitent à une modification de plusieurs techniques ou systèmes exploités au quotidien. Force est de constater que l'évolution technologique et l'insertion de nouveaux matériaux exploratoires dans la démarche artistique de nombreux artistes sont concomitantes aux transformations qui bouleversent le fondement et les lignes directrices de la conservation préventive ou curative dans sa définition traditionnelle, lignes à reconsidérer en fonction de l'actualisation des médias, de la multiplicité des supports et, conséquemment, de leur caractère obsolescent qui outrepasse la question de contrôle environnemental ${ }^{[2]}$.

Les stratégies abordées concernant la protection de l'information contenue dans l'œuvre et de son support remettent inévitablement en question les notions traditionnelles de conservation, demandent l'adoption de nouvelles politiques culturelles et tendent à être appliquées dans toutes les divisions du terrain muséal. Les archivistes des collections sont doublement concernés par ce nouvel héritage. Certes, il leur faut inventorier, gérer et documenter l'information de base, mais également réfléchir à une révision constante de leurs dispositifs de travail, afin d'en arriver à produire un système commun et malléable, le paradigme de la préservation appelant nécessairement à une progression en simultané avec les technologies.

À l'ère des possibilités et d'une conservation exemplaire de l'œuvre dite traditionnelle, acquise en partie et paradoxalement grâce aux développements technologiques menant à l'utilisation de procédés novateurs en matière de restauration, de nouvelles stratégies emboîtent le pas. On ne parle donc plus de conservation "à l'identique» " ${ }^{(3)}$, mais plutôt d'émulation, de migration et de ré-interprétation. Ces procédés proposant la recherche d'équivalences par l'imitation de l'apparence d'origine, par la mise à niveau ou par la recréation de l'œuvre suscitent bien des débats autour de l'esthétique initiale, de la 
valeur intrinsèque et des questionnements au sujet de la propriété intellectuelle. Alors que certains approuvent, d'autres s'opposent aux potentialités de la migration, qui risque trop souvent de modifier la forme de l'œuvre, tout comme à la ré-interprétation et aux transmutations substantielles qu'elle provoque.

Les pratiques multidisciplinaires adoptées par les artistes en art contemporain se transforment et bouleversent les standards de conservation jusqu'alors clairement établis. Ces mutations engendrent des questionnements différents auxquels le musée doit répondre, sous peine de voir se perdre les premiers signes, les premières interventions et créations artistiques employant des composantes technologiques. Dans ce cas précis, la ligne du temps présente des variations et des modifications majeures qui gagnent à être étudiées et reconsidérées à la vitesse de leurs changements. Sans y aller d'une rupture, il s'agirait de voir comment on peut établir une cohésion entre les structures usuelles et les nouvelles interrogations que soulève la conservation. Par ailleurs, le regard posé aujourd'hui sur le musée d'art contemporain, qui accueille essentiellement des artistes vivants, nous invite à repenser le statut de l'artiste en tant qu'acteur décisif au centre de ce processus. Mon expérience à titre de stagiaire aux Archives des collections du Musée d'art contemporain de Montréal (MACM), sous la supervision de madame Anne-Marie Zeppetelli, archiviste des collections, m'a permis de revoir les différentes sources théoriques, d'échanger avec les experts soucieux de la pérennité de ces œuvres et, surtout, de constater, sans aucune équivoque, la portée de la documentation sur la conservation ${ }^{[4]}$.

Cette analyse n'entend pas présenter de conclusions formelles à l'égard des pratiques à adopter, mais s'interroge plutôt sur les potentialités d'une méthodologie d'assise, basée sur un questionnaire de conservation et sur l'importance de soutenir un entretien avec l'artiste. Dans le même souci de logique, nous ne saurions évoquer cette question sans traiter des acteurs qui ont à composer avec le sujet qui nous intéresse. En tirant profit des pratiques déjà en place et des expériences vécues par les professionnels de la conservation, on pourra déterminer plus aisément les outils, les actions, ainsi que les spécialistes les mieux qualifiés pour prendre des choix éclairés.

\section{[4]}

Au MACM, près de $90 \%$ de la collection permanente peut être conservée avec les ressources et les méthodes traditionnelles de conservation, ce qui diffère pourtant d'un certain pourcentage d'œuvres photographiques réalisées à partir de technologies plus récentes, d'œuvres vidéographiques (environ 200 œuvres datant de la fin des années 1970 et du début des années 1980) ou d'œuvres employant des médias variables (plus de 25). La connaissance de la variabilité des composantes devient donc primordiale. On en a vu les premières conséquences avec l'acquisition courante de bandes sonores et magnétiques dans les années 1960 et 1970 , dont on connaît aujourd'hui davantage la fragilité.

[5]

BUREAUD et al., Art et technologie : la monstration, p. 181.

\section{Définition, comportements et caractéristiques}

Une forme de compréhension historique, suggérée dans le rapport intitulé Art et technologie : la monstration, applique la notion de "strates temporelles " ${ }^{[5]}$, cette dernière faisant foi de l'étendue de l'histoire des œuvres électroniques 
qui, bien que récente, peut être répartie à l'exemple du modèle classique; on peut y distinguer trois «âges " distincts :

l'art en création, actuel, en train de se faire; l'art récent, qui a déjà quelques années d'existence, ne pose pas de problèmes techniques particuliers de monstration, mais a déjà subi un premier filtre de validation et de reconnaissance (Legible City de Jeffrey Shaw en est le parfait exemple); l'art "ancien ", il appartient déjà à l'histoire de l'art électronique et il peut commencer à présenter des difficultés dans la monstration ${ }^{[6]}$.

Dans ce contexte, et en raison de la configuration éphémère des

Pour la définition, voir DICTIONNAIRE DES ARTS MÉDIATIQUES.

Multimédia. <http://www. comm.uqam.ca/\%7EGRAM/ frames/termM.html (consulté en mars 2006).

[9]

Pour la définition, voir DICTIONNAIRE DES ARTS MÉDIATIQUES Art médiatique. <http://www. comm.uqam.ca/\%7EGRAM/ frames/termA.html> (consulté en mars 2006)

[10]

$B A L P E$, Jean-Pierre, 1999, p. 65, cité dans FOURMENTRAUX, Jean-Paul. Art et Internet. Les nouvelles figures de la création. Paris : CNRS Communication, 2005, p. 176. dispositifs générant ces œuvres, il paraît important de considérer ces trois modes de distinction ou «strates » entre les différentes périodes de création de l'art. En proposant une réflexion sur le temps et l'espace, cette perspective insère distinctement l'œuvre dans sa jeune réalité historique et distingue l'art "ancien" des formes et des systèmes communicationnels émergents.

Au-delà de la documentation, l'art technologique requiert une connaissance des équipements techniques et un contrôle des règles de fonctionnement, savoir qui s'avère indispensable à une meilleure compréhension des systèmes générateurs et de leurs comportements, qu'ils soient analogiques ou numériques, mécaniques ou électroniques, interactifs ou non. Si l'on reprend la division établie par le Réseau des médias variables, les œuvres à composantes technologiques peuvent être représentées par huit caractéristiques spécifiques : contenue, installée, performée, interactive, reproduite, dupliquée, encodée et en réseau ${ }^{[n}$. Sans dresser un portrait fixe - ce dernier requérant un suivi dans le temps -, nous nous sommes interrogée sur la

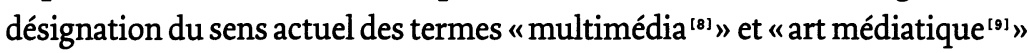
pour les regrouper.

Par ailleurs, l'orientation et les grandes tendances de tels dispositifs artistiques peuvent être dépeintes par la variété des sujets traités, par l'hybridité des matériaux et par les nombreuses zones d'exploration possibles qu'elles suggèrent :

l'œuvre d'art, aujourd'hui, tend à prendre la posture "financière", celle du flux où l'œuvre n'a plus de valeur en tant que telle mais où cette valeur n'est que dans la captation, de préférence inépuisable, des déplacements incessants qu'elle provoque : '’euvre n'est plus dans l'objet mais dans les possibilités de l'objet, l'« avoir " a moins d'importance que le «saisir». Le "produire» n'est plus dans le " reproduire» mais dans le «re-produire : l'œuvre d'art est dans l'événement toujours recommencé dont on ne peut conserver que des traces ${ }^{100}$. 
Ce passage illustre bien les schèmes de conception dans lesquels résident la marche de l'œuvre et l'idée de transformation, deux concepts qui influent et agissent tant sur le plan de la monstration que celui de la conservation. Dans les deux cas, la préoccupation centrale demande une réflexion sur les dispositifs de présentation valables lors d'une exposition, l'aspect conservation devant intervenir tout au long de la mise en réserve et plus spécialement comme étape préliminaire à une présentation.

Le Net art, tout comme les autres formes d'art issues des caractéristiques énoncées plus tôt, pose à l'avant-plan des problèmes de conservation relatifs à la saisie des modifications ou des changements intermédiaires engrangés par une action, par une présentation de l'œuvre dans un autre cadre ou par une problématique de conservation ayant obligé une quelconque forme de mutation qui déroge de l'esthétique initiale.

Dans son ouvrage sur le Net art, Jean-Paul Fourmentraux décrit cette nouvelle dimension de l'œuvre du Net art et de la navigation, dont le processus flexible émerge au-delà d'une structure homogène et cohérente et où la création de l'artiste-auteur et des collaborateurs informatiques engendre une multitude de possibilités, de dynamiques et de fragments modifiables et transposables qui, par la manifestation et l'immersion des visiteursacteurs, génère un réseau ouvert, marqué par des passages successifs et une démultiplication du travail personnel de l'auteur, dérivée d'une appropriation collective des publics et d'une hybridation leur permettant d'évaluer le réel autrement ${ }^{[11]}$.

Conservation traditionnelle vs conservation d'œuvres

à composantes technologiques

Le phénomène de dissociation entre les stratégies de conservation classiques déjà en place et celles qui tendent à ressortir en raison de la spécificité des composantes technologiques résulte principalement de l'écart entre le temps d'intervention requis par une œuvre historique depuis le moment de sa création et la désuétude en version accélérée des œuvres dont il est ici question ${ }^{[12]}$. Selon Hervé Fischer, artiste-philosophe, la diffusion et la conservation des œuvres historiques, qu'il désigne "de premier mode " ${ }^{[13]}$, n'est pas archaïque - ce qu'elle aurait pu laisser entrevoir -, mais bien durable :

Le contenu d'un disque optique se conserve beaucoup moins longtemps (une dizaine d'années environ) que celui d'un livre papier, d'une bobine de film $35 \mathrm{~mm}$, d'un des manuscrits de la Mer morte, voire que celui des peintures préhistoriques de la grotte de Chauvet (datant de 32000

\section{[11]}

Idem, p. 180-181.

[12]

On sait que les musées appliquent globalement des stratégies de conservation préventive découlant entre autres du suivi de guides de conservation arborant les meilleures pratiques à observer comme la prise en compte de facteurs tels que l'entreposage des œuvres dans un environnement stable et contrôlé, des procédures de manutention adéquates, des plans d'action en cas de sinistre ou des mesures de sécurité. Toutes ces normes restent inévitablement à considérer.

[13]

HORIZON ZÉRO.

Mémoire : les amnésiques numériques. Réflexions sur la fragilité de la mémoire numérique. <http://www. horizonzero.ca/textsite/ghost. php?is $=18 \&$ file $=6$ \&tlang $=1>$ (consulté en mars 2006). 
Une révision des schèmes de documentation et une évaluation des stratégies de conservation plus récentes comme l'émulation ou la migration par le biais d'études de cas, à l'exemple de l'exposition Seeing Double: Emulation in Theory and Practice organisée au printemps 2004 par le Solomon R. Guggenheim Museum, en partenariat avec

la FDL, permettraient d'adapter de façon complémentaire les méthodes déjà en place. Et puisque le droit moral de l'artiste lui donne autorité sur le choix final des moyens à privilégier, il serait juste d'en connaître les avantages et les contraintes. Voir à ce sujet RÉSEAU DES MÉDIAS

VARIABLES. Seeing Double Emulation in Theory and Practice.

$<$ http://www.variablemedia net/f/seeingdouble $>$ (consulté en février 2006)

[ 7$]$

Dans cet extrait des actes du $30^{\mathrm{e}}$ congrès de l'Association des archivistes du Québec, l'auteure se penche sur la notion d'impact du numérique sur les normes et les supports traditionnels de préservation. Elle soumet différents tableaux qui établissent une comparaison entre les modes de préservation traditionnelle et numérique en abordant la préservation de la qualité, de la longévité, de l'intégrité physique et intellectuelle, du choix de substitution ou de transfert, de l'accessibilité et des exigences de la technologie numérique. Voir CENTRE DE CONSERVATION DU QUÉBEC. Impact du numérique sur les normes et les supports traditionnels de préservation. <http://www.ccq.mcc. gouv.qc.ca/formulaires/impact_ numerique.pdf $>$ (consulté en mars 2006) ans et encore intactes!) ou que les empreintes de pas dans la glaise de trois hominidés, deux adultes et un enfant (qui datent de 3,6 millions d'années, Laetoli, Tanzanie). Aux amateurs de photos numériques, il faut le rappeler : il serait sage d'imprimer vos photos sur du papier... ${ }^{[14]}$.

Les professionnels de la conservation ont toujours eu à s'adapter à l'apparition de nouveaux matériaux ou médiums privilégiés et consommés par les artistes à une période donnée. Néanmoins les œuvres " de second mode» " ${ }^{[15]}$ impliquent des corps technologiques toujours plus vulnérables et éphémères, qui réagissent différemment et interagissent entre eux, leur variabilité allant au-delà des réactions physiques ou des facteurs de dégradation relativement contrôlables. Car, même dans un environnement protégé, ces composantes se voient supplantées par rapport aux technologies naissantes toujours plus innovatrices et performantes, leur entreposage prolongé, même durant une brève période, risquant de créer des surprises à la sortie.

Comment déceler les changements avant même qu'ils ne surviennent, évitant ainsi qu'un système flanche ou ne devienne léthargique? Et si ces œuvres étaient condamnées à paraître de façon passagère, puis à séteindre avec les traces significatives de matériaux trop hâtivement révolus ou à être présentées de façon fragmentaire, avec des bribes d'information et un disfonctionnement des systèmes qui les régissent? L'exposition partielle ou intégrale de ces coquilles vides ne restituera jamais ne serait-ce qu'un aperçu de ce qu'elles étaient en réalité, la conservation de l'environnement ou du logiciel immatériel, les données de l'œuvre et son comportement l'emportant sur l'apparence matérielle. Une installation vidéographique interactive résidant dans un code, à l'exemple de Générique d'Alexandre Castonguay (Collection Musée d'art contemporain de Montréal, 2001), ne pourrait être pensée autrement qu'avec la présentation de son environnement immatériel. Dans tous les cas, cela implique une constance en matière de documentation. De là l'importance de nous repositionner et d'instaurer des pratiques propres à ces collections à risque, inspirées des acquis, mais évidemment renouvelées par l'investigation des technologies et de leur historique, par l'observation et la consignation des changements signifiants qu'elles engendrent, par l'expérimentation de traitements individualisés (études de cas) ${ }^{[16]}$ et de mécanismes favorisant leur mise en valeur, étapes qui pourraient répondre aux interrogations que leur sauvegarde et leur transmission future suscitent.

Avant de passer à l'étude des stratégies envisagées actuellement par le réseau, il semblait pertinent de dresser un portrait comparatif de la conservation des œuvres d'art historiques par opposition à des œuvres à composantes technologiques. Pour ce faire, l'étude réalisée par Francine Gauthier pour le Centre de conservation du Québec (CCQ) se révèle fort à propos ${ }^{[1]}$. 
Pour l'heure, nous laisserons de côté les modes de l'archivage de l'information, pour ne retenir que certains points consacrés à la conservation, en les adaptant aux modes de conservation des œuvres à composantes technologiques ou à certains éléments comparables :

\section{TABLEAU 1}

Tableau de conservations comparées

\begin{tabular}{|c|c|}
\hline EUUVES TRADITIONNELLES & EUUVES À COMPOSANTES TECHNOLOGIQUES \\
\hline $\begin{array}{l}\text { - Préoccupation tournée vers la réversibilité } \\
\text { des procédés de restauration }\end{array}$ & $\begin{array}{l}\text { - Préoccupation tournée principalement vers la migration } \\
\text { et l'émulation }\end{array}$ \\
\hline $\begin{array}{l}\text { - Conservation à l'identique privilégiée qui tend à } \\
\text { empêcher la perte de valeur de témoignage }\end{array}$ & $\begin{array}{l}\text { Conservation partielle de l'œuvre originale par l'imitation de } \\
\text { son apparence d'origine ou par des modifications notables } \\
\text { obligées par sa mise à niveau }\end{array}$ \\
\hline $\begin{array}{l}\text { - Contrôle efficace de la longévité sur les processus } \\
\text { de conservation préventive et curative }\end{array}$ & $\begin{array}{l}\text { - Contrôle partiel de la longévité par l'attention aux modes } \\
\text { de conservation préventive traditionnelle, mais rhizomique } \\
\text { quant à l'impact réel des nouvelles stratégies de restau- } \\
\text { ration envisagées }\end{array}$ \\
\hline $\begin{array}{l}\text { - Méthodes connues de stabilisation et de limitation } \\
\text { des effets qui causent la dégradation }\end{array}$ & $\begin{array}{l}\text { - Incertitude quant au maintien et à l'entretien de l'équipe- } \\
\text { ment technologique produit sur le marché, due à l'absence } \\
\text { de contrôle sur l'évolution des technologies générées } \\
\text { par l'industrie }\end{array}$ \\
\hline
\end{tabular}

Déjà, en 1991, Jan Michaels de la Bibliothèque nationale du Canada divulguait les résultats d'une étude exposant les problèmes techniques des enregistrements sonores (rubans de magnétophones à bobines, cylindres, disques longue durée, 45 tours et 78 tours) conservés par l'établissement et questionnait la possibilité d'assurer leur conservation en effectuant un "relogement » ou des copies de conservation. Les tests effectués et les renseignements obtenus annonçaient des difficultés du point de vue de leur conservation, tant sur le plan technique qu'éthique : " Ainsi, sur le plan éthique, la question demeure à savoir si la réparation du médium doit d'abord viser à obtenir une audition supplémentaire de l'enregistrement ou à assurer sa conservation pour la postérité ${ }^{183}$."

Depuis, les avenues qu'offrent des stratégies comme l'émulation et la migration apparaissent incontournables, même sila polémique entourantle plan éthique reste un fort sujet d'actualité. L'émulation permet d'imiter l'apparence d'origine d'une œuvre. La substitution des composantes impliquerait,
[18]

MICHAELS, Jan.

"The Condition Survey of Sound Recordings at the National Library of Canada: Implications for Conservation *, Sauvegarder le $X X^{e}$ siècle: la conservation des matériaux modernes. Ottawa, Canada : Institut canadien de conservation, 1993, p. 21. 
[19]

RÉSEAU DES MÉDIAS VARIABLES. Seeing Double

Emulation..., op. cit.

[20]

TATE ONLINE.

Major Projects. <http:// www.tate.org.uk/research/ tateresearch/majorprojects/ mediamatters/resource_installationdoc.htm $>$ (consulté en mars 2006)

[21]

Voir, entre autres : INTERNATIONAL NETWORK FOR THE CONSERVATION OF CONTEMPORARY ART (INCCA). Guide to Good Practice: Artists' Interviews. বhttp://resourceguide. eai.org/collection/computer/ pdf/incca.pdf> (consulté en

mai 2007) ; ainsi que TATE RESEARCH. Research projects. Interviews with Artists. <http://

www.tate.org.uk/research/ tateresearch/majorprojects/ interviews.htm $>$ (consulté en mars 2006)

[22]

RÉSEAU DES MÉDIAS VARIABLES. Seeing Double

Emulation..., op. cit.

D'autres organisations méritent d'être considérées. Parmi ces approches, voir, notamment,

l'Art Gallery of Western

Australia, le Banff New Media Institute, la Société des arts

technologiques, le Walker

Art Center, l'Ars Electronica

(qui dispose d'un des plus importants centres d'archive dédiés à l'art numérique) et le V2_ Institute for the Unstable Media (centre interdisciplinaire consacré à l'art et à la technologie médiatique).

[23]

COLARDELLE, Michel.

"Le conservateur et la recherche". Introduction à la muséologie MSL-1896. Recueil de notes. Université

Laval, Les Presses de I'Université Laval, 1999, p. 84. par exemple, de réutiliser sans conversion le code ou les programmes écrits d'un logiciel pour un ordinateur en le transférant dans un autre. La migration, quant à elle, demanderait de passer d'un équipement à un autre, par une mise à niveau vers une version plus récente et, conséquemment, différente. Faute de conserver l'œuvre à l'identique - le stockage de matériaux et d'équipements dans les réserves ne suffisant plus en raison de leur caractère éphémère ${ }^{[19]}$-, ces solutions procurent aux artistes et aux musées une capacité de re-présentation des œuvres, en permettant aux dispositifs qui les commandent et les génèrent de fonctionner à nouveau.

\section{L'approche actuelle des musées : pratiques et stratégies}

L'absence de structures rigoureuses et de protocole d'intégration spécifique aux œuvres à composantes technologiques entraîne des écarts considérables quant à la classification et des lacunes liées à la terminologie employée. La documentation actuelle s'en tient, le plus souvent, aux champs traditionnels et laisse suggérer que les musées acquièrent en grande partie ces œuvres selon les mêmes procédures, en étant plus ou moins conscients des obstacles qu'elles représentent.

Sur un autre plan, de nombreuses organisations contribuent activement à leur documentation, à leur conservation et à leur diffusion. Au Tate Modern Museum, les œuvres nouvellement acquises de la collection d'art contemporain passent par un entretien détaillé entre le conservateur qui s'appuie sur un guide ${ }^{[201}$ - et l'artiste ${ }^{[211}$. Une autre initiative majeure demeure celle de Jon Ippolito, conservateur associé des arts médiatiques au Solomon R. Guggenheim Museum. La création du paradigme des médias variables et les réflexions menées par le biais du Réseau des médias variables du Guggenheim en partenariat avec la fondation Daniel Langlois pour l'art, la science et la technologie reposent d'ailleurs sur la conservation d'œuvres réalisées à l'aide de médias non traditionnels. À ce jour, le réseau a déjà investi dans de multiples recherches; au centre du projet, le Questionnaire sur les médias variables ${ }^{[22}$.

\section{Rôles et responsabilités}

Dans la pratique, «les conservateurs du patrimoine exercent des responsabilités scientifiques et techniques visant à étudier, classer, conserver, entretenir, enrichir, mettre en valeur et faire connaître le patrimoine » ${ }^{[23}$. Pour reprendre 
les propos de Jan Michaels concernant la problématique de conservation des enregistrements sonores à la Bibliothèque nationale du Canada, on constate que la pérennité des œuvres peut être discutée de différentes façons. Sur ce point, deux conceptions s'affrontent. Viser une audition supplémentaire de l'enregistrement ou une mise à niveau de l'équipement par le transfert aux écrans plats d'une vidéo-sculpture - par exemple En attendant la pluie de Michèle Waquant (Collection Musée d'art contemporain de Montréal, 1987) - semble, pour certains conservateurs et à juste titre, une solution peu acceptable. Il s'agirait alors d'assurer autant que possible la conservation des vidéogrammes couleur et des moniteurs en procédant éventuellement à l'acquisition et au stockage de plusieurs moniteurs identiques. Cette stratégie représente néanmoins des coûts qui ne garantissent en rien le fonctionnement à long terme du matériel de «rechange ».

La seconde conception oblige certes la mise en application de stratégies plus à risque comme la migration, mais assure, lorsque autorisée par l'artiste, une existence prolongée à l'œuvre. Reconduite de cette façon, on pourrait faire état, lors des futures présentations, de son passage d'un support ou d'un équipement à un autre. Le défaut de ces nouvelles formes de conservation ou de restauration réside dans leur caractère transmuable, où l'œuvre sera appelée à évoluer de génération en génération. Le danger serait d'en altérer profondément la nature, d'où l'importance d'en conserver les traces et de capturer, a priori, l'intention de l'artiste. Une des solutions tient peut-être dans la substitution des composantes, astuce qui peut permettre, si l'on pense à I Can't Hear You (Autochthonous) de Tony Oursler (Collection Musée d'art contemporain de Montréal, 1995), de faire fonctionner l'œuvre sans nuire à son esthétique, alors qu'en réalité la conception d'une régie dissimulant un lecteur DVD procure l'impression aux visiteurs que le lecteur VHS disposé devant l'œuvre demeure toujours opérationnel.

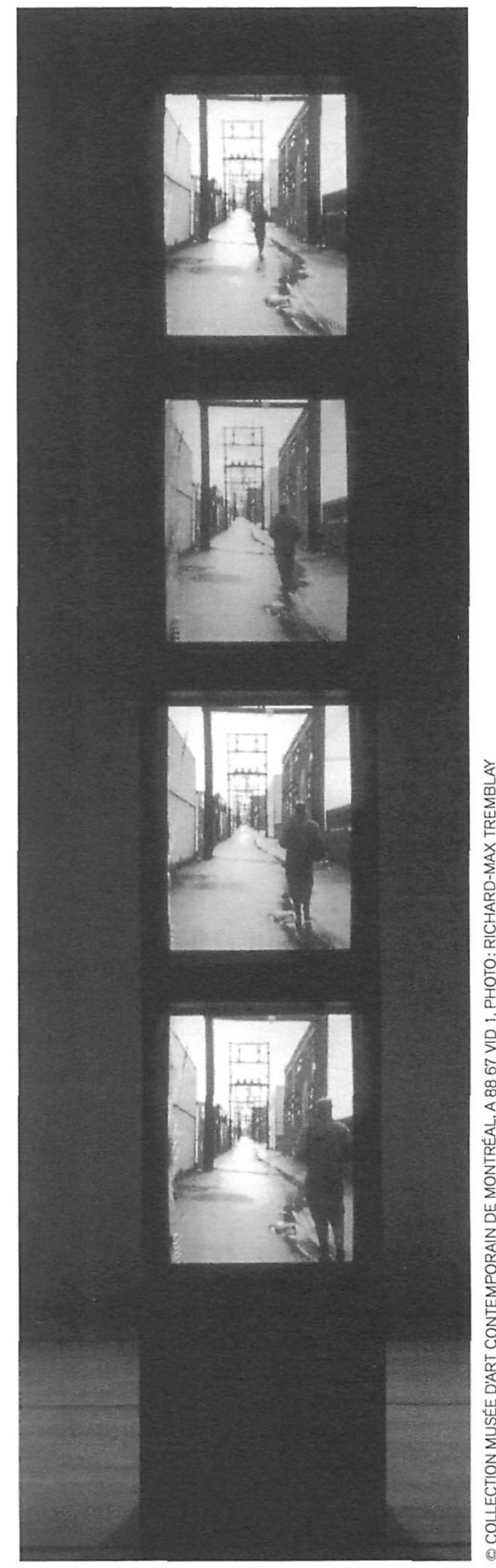

FIGURE 1

Waquant, Michèle

En attendant la pluie/

Waiting for the Rain, 1987

4 moniteurs, support, 4 vidéo-

grammes couleur, $11 \mathrm{~min}$., son,

$315 \times 51 \times 49.5 \mathrm{~cm}$ 
[24]

Pour citer Jon Ippolito: « lls [les artistes et les institutions] pourraient commencer à envisager une œuvre durable non pas comme une relique de pierre - car la pierre s'effrite -, mais plutôt comme une succession d'événements interdépendants qui, à l'instar d'un cours d'eau subsistent en demeurant variables." (IPPOLITO, Jon. "Composer avec l'imprévisible: le questionnaire sur les médias variables", L'approche des médias variables, la permanence par le changement. New York : Guggenheim Museum Publications, 2003, p. 53.)

[25]

Document de travail non employé par le MACM actuellement.

[26] Idem

[27]

Ce champ requiert des spécifications sur le concept et la démarche artistique, l'identification avec un haut degré de précision des composantes, la description des étapes d'installation au regard des aspects propres aux composantes technologiques, ainsi que l'identification des formats originaux (audio, vidéo, etc.)
Dans un musée comme le MACM, le Département des archives des collections se chargera de saisir toute information nécessaire à la base de données. L'archiviste prendra ensuite soin de déterminer avec l'aide du restaurateur l'emplacement approprié de l'œuvre dans la réserve. Les composantes non acquises avec l'œuvre pourront quant à elles être entreposées au Département de l'audiovisuel. En cas de re-présentation ultérieure en salle, le conservateur à la collection, l'archiviste des collections et le restaurateur assumeront généralement les décisions à prendre en termes de restauration. Du coup, ce mode de fonctionnement exige plus d'un intervenant et s'accompagne, lorsque possible, du verdict de l'auteur.

Si la documentation et le travail avec l'artiste font partie intégrante du rôle d'un conservateur, le manque de temps et des pratiques conditionnées par les budgets font foi de dossiers parfois vides ou incomplets. Rester à l'étape de l'entreposage et de la conservation traditionnelle serait ignorer la complexité de telles œuvres et les voies novatrices qui s'ouvrent aux professionnels des musées. Il devient dès lors primordial de réfléchir sur le rôle et les responsabilités du conservateur dans le processus de recherche et de documentation, en examinant les facteurs qui guideront ses choix de conservation ${ }^{[24]}$.

\section{Stratégies à privilégier}

L'état actuel des stratégies qu'on nous propose demande une méthode ad hoc axée sur la documentation. À l'inverse des données rassemblées jusqu'à présent en art contemporain, le dossier de l'œuvre devrait inclure des considérations plus exhaustives sur le parcours et la nature des transformations apportées.

Puisque l'introduction d'un questionnaire apparaît comme l'un des objets centraux de cette analyse, il convient de présenter à ce stade un survol des moyens d'investigation élaborés au MACM (Questionnaire pour les œuvres à composantes technologiques ${ }^{[25]}$ et Fiche de catalogage ${ }^{[26]}$ ) en fonction des œuvres de médias variables à composantes numériques, audiovisuelles et sonores de la collection permanente d'œuvres d'après 1980 du Musée et à partir desquelles nous avons pu saisir un corpus et esquisser des outils indispensables aux problèmes que nous connaissons.

Divisé en quatre zones principales, le questionnaire s'articule autour de variations qui présentent un intérêt particulier dans l'étude qui nous concerne : 
1] Renseignements sur l'œuvre et l'artiste

2] Description technique ${ }^{[2]]}$

3] Zone de description technique pour les œuvres numériques

4] Zone de description technique pour les œuvres interactives

5] Consentement relatif à la conservation préventive et à la restauration

6] Consentement relatif à la reproduction pour des fins de conservation

\section{Mise à jour du questionnaire (cycle de rafraîchissement)}

Ce questionnaire demande de reconnaître et d'approfondir tout autant les notions relatives aux médias souvent discontinus et provisoires, aux formats des composantes, au langage de programmation, au type de logiciel employé ou à tout élément immatériel, ainsi que leur intégration explicite, afin que l'artiste puisse y répondre, le cas échéant. Il incombe donc, si l'on veut obtenir des résultats éloquents et, surtout, utiles à la prise de décisions ultérieures, de poser les bonnes questions et d'intégrer, aux schèmes de documentation, l'application d'un questionnaire qui correspond à la nature unique de ces œuvres ${ }^{[28]}$.

L'emploi d'une fiche de catalogage comportant des champs en interrelation avec le questionnaire de conservation joue un rôle complémentaire. La version réinterprétée à partir de la fiche initiale du MACM considère certains paramètres attenants tels que la mention, dans le champ "ARTISTE", du nom du détenteur du droit d'auteur et du représentant ${ }^{[29]}$ et, dans le champ "DESCRIPTION DE L'๕UVRE", des données compatibles au corpus sélectionné, de même qu'aux caractéristiques développées par le Réseau des médias variables : 1) « POUR LES (EUVRES REPRODUITES ${ }^{(30)}$ ), 2) « POUR LES @EUVRES DUPLIQUÉES ${ }^{[31}$ ) et 3) « POUR LES đEUVRES ENCODÉES ${ }^{1321}$ ». Enfin, les champs « EXPOSITTON(s)» et «ÉTAT DE CONSERVATION » exigent tout autant d'indications sur les variations de l'œuvre lors de chaque présentation.

Dans cette perspective, l'importance d'une action immédiate dirigée vers le conservateur, l'archiviste et le restaurateur s'impose d'elle-même. On ne peut plus ignorer la coopération avec l'artiste, mais également avec le collaborateur qui contribue à la mise en œuvre de la création (dans le cas de Castonguay, cela impliquerait l'apport du programmeur Mathieu Bouchard dans le processus de documentation et de conservation). On ne le dira jamais assez, les champs très techniques de ces œuvres, qu'elles soient

\section{[28]}

Autrement, ce serait méconnaître les œuvres et sous-estimer les difficultés qui pourraient poindre à l'horizon. Richard Rinehart, directeur des médias numériques au Berkeley Art Museum / Pacific Fil Archive, précise que la documentation actuelle ne peut assurer, à elle seule, la capacité à répondre aux environnements complexes des éléments multimédias : "the radical intentionality encapsulated in their form is also in danger of being diluted as museums inappropriately apply traditional documentation and preservation methods or ignore entire genres of these works altogether ". (RINEHART, Richard. A System of Formal Notation for Scoring Works of Digital and Variable Media Art. Berkeley : University of California, s.d., p. 2-3.)

\section{[29]}

Ce champ implique la saisie d'information sur le galeriste ou sur la personne chargée de la gestion du droit d'auteur. Pensons aux distributeurs d'artistes vidéographiques tels que Vidéographe, Vitape et Electronics arts.

[30]

Ex. : format audio et/ou photo original, format film et/ou vidéo ou support d'enregistrement original, format original d'impression et format de la(des) copie(s) d'exposition.

[31]

Ex. : composante(s) duplicable(s).

[32]

Ex. : langage de programmation ou système de notation symbolique, environnement auteur ou type de logiciel, système de compression du son et vidéo employé, rapport de format et code non standard. 
Conversation avec M. Richard Gagnier, conservateur des œuvres d'art contemporain au

MBAC

[34]

Idem.

audiovisuelles, holographiques, numériques, sonores, vidéographiques, etc., obligent une connaissance plus que fragmentaire, mais bien approfondie des appareils technologiques et de leurs comportements. Or, on sait aujourd'hui qu'une immense partie du problème réside dans cet aspect. Un conservateur, qui pourtant connaît bien sa collection, n'est pas forcément à l'affût de l'ensemble des paramètres des œuvres qu'il représente et dont il a la charge. Doit-on penser à recruter dans les musées des conservateurs technologues? Nous ne le pensons pas. La plupart des conservateurs ne sont ni programmeurs, ni concepteurs de logiciels, ni analystes de linformatique, mais historiens de l'art. Au-delà d'une maîtrise technique intrinsèque aux matériaux du multimédia, le rôle du conservateur suppose a priori une connaissance scientifique de l'œuvre d'art, tant esthétique qu'historique, des différentes manifestations artistiques et des méthodes de travail analytiques et critiques de l'histoire de l'art. Cela dit, la principale difficulté tient du fait que l'artiste demeure maître du choix des composantes qu'il intègre à sa création et avec lesquelles le musée aura à composer.

Tel que constaté au MACM, le rôle occupé par le Département des archives des collections est substantiel. Le conservateur, l'archiviste des collections et le restaurateur travaillent en interrelation, si bien que l'application d'un questionnaire de conservation solliciterait et consoliderait l'apport de ces trois autorités. Pour les musées, l'une des solutions à privilégier reste avant tout la collaboration, même si chacun sait que l'artiste doit être avisé lorsqu'une intervention majeure est à prévoir sur son œuvre.

Selon Richard Gagnier, conservateur des œuvres d'art contemporain au Musée des beaux-arts du Canada (MBAC), son institution a pour sa part instauré, au fil des années, un mode de fonctionnement efficace, résultant d'une bonne collaboration entre le conservateur, le restaurateur et les techniciens du service multimédia :

Le protocole d'intégration des œuvres à composantes technologiques est partie prenante du processus d'acquisition de l'œuvre. Bien que la procédure ne soit pas systématique dans la manière de l'appliquer, le processus exige de la part du conservateur intéressé par l'achat d'une telle œuvre de discuter avec les gens du service multimédia, d'abord des exigences de l'œuvre en termes d'équipements; ces équipements devront être achetés ou non dépendant de l'œuvre et des ententes avec l'artiste et du marchand ${ }^{\text {[33]. }}$.

L'approche appliquée - les œuvres étant installées, avant l'acquisition, « afin de vérifier le fonctionnement et les exigences d'installation (dimensions de la salle, atmosphère, éclairage, couleurs sur les murs et plafond, construction d'une salle technique attenante pour les équipements, etc.) )" ${ }^{[34}$ - pourrait donc être reprise de façon plus systématique par les musées. 


\section{Conclusion}

Au regard de cette analyse, il paraissait pertinent d'énoncer une série de recommandations - ces procédures ayant avantage à découler d'un protocole d'intégration - à partir de repères, certains existants, transférables aux œuvres à composantes technologiques :

- considérer les politiques en vigueur dans d'autres institutions;

- favoriser les échanges, dans le but de profiter largement des ressources et des expertises intéressées et concernées par la problématique;

- viser une certaine interopérabilité des mesures employées entre les institutions concernées;

- accroître la collaboration entre les professionnels habilités à la conservation des œuvres au sein de l'institution;

- redéfinir les protocoles d'intégration en les adaptant aux œuvres à composantes technologiques;

- s'il n'y a pas de collection existante, dresser un inventaire des œuvres et les réunir au sein d'une même collection;

- envisager l'utilisation d'outils permettant une approche et une conservation appropriées de l'œuvre (questionnaire de conservation, entretien avec l'artiste suivant une grille préétablie);

- procéder à des études de cas afin d'évaluer l'application possible de nouvelles stratégies de conservation (ex. émulation, migration);

- effectuer la mise à jour des œuvres tous les cinq à sept ans ${ }^{\text {[35] }}$ en les soumettant à une épreuve de contrôle;

- observer les changements signifiants dus à l'évolution technologique, afin de s'assurer du «bon fonctionnement» des œuvres;

- assurer et compléter la documentation des œuvres de la collection (ce qui requiert une documentation en continue par l'actualisation des variations de l'œuvre);

- etc.

Sans avoir résolu l'ensemble des interrogations soumises en introduction, l'application d'une méthode d'investigation à l'exemple du Questionnaire pour les œuvres à composantes technologiques réintroduit la place de la collaboration entre les professionnels de la conservation de l'art contemporain ou

[35]

Un logiciel comme Générique d'Alexandre Castonguay, semble assez robuste pour ne pas poser de réels problèmes de conservation pendant environ dix ans. Voir au bon fonctionnement des œuvres selon une période donnée (ex. tous les cinq ans) pourrait donc constituer une approche efficace. 
[36]

Ce graphique est tiré de VEILLEUX, Cindy. La conservation des œurres à composantes technologiques. Essai (muséologie). Québec : Université Laval, 2005-2006. les responsables de collections muséales et l'artiste, processus devant être soutenu dès l'acquisition et maintenu par un dialogue entre les acteurs, et qui gagnerait à être appliqué au sein des musées concernés. Une collaboration encore plus étroite, avec l'artiste et son collaborateur, suffirait également à annoncer les grandes lignes des décisions qui devront être prises à court et à long terme. Il convient donc de réfléchir à la possibilité de soutenir plus avant ces liens. La représentation modélisée ci-dessous illustre cette proposition en positionnant le questionnaire - ce dernier engageant à la saisie des données exposées plus tôt -, l'artiste et l'œuvre au centre des principaux acteurs (le conservateur, l'archiviste, le restaurateur et le technicien), qui auront à assumer un rôle déterminant dans la procédure de conservation.

\section{DIAGRAMME 1}

Dialogue entre les acteurs de la conservation ${ }^{[36]}$

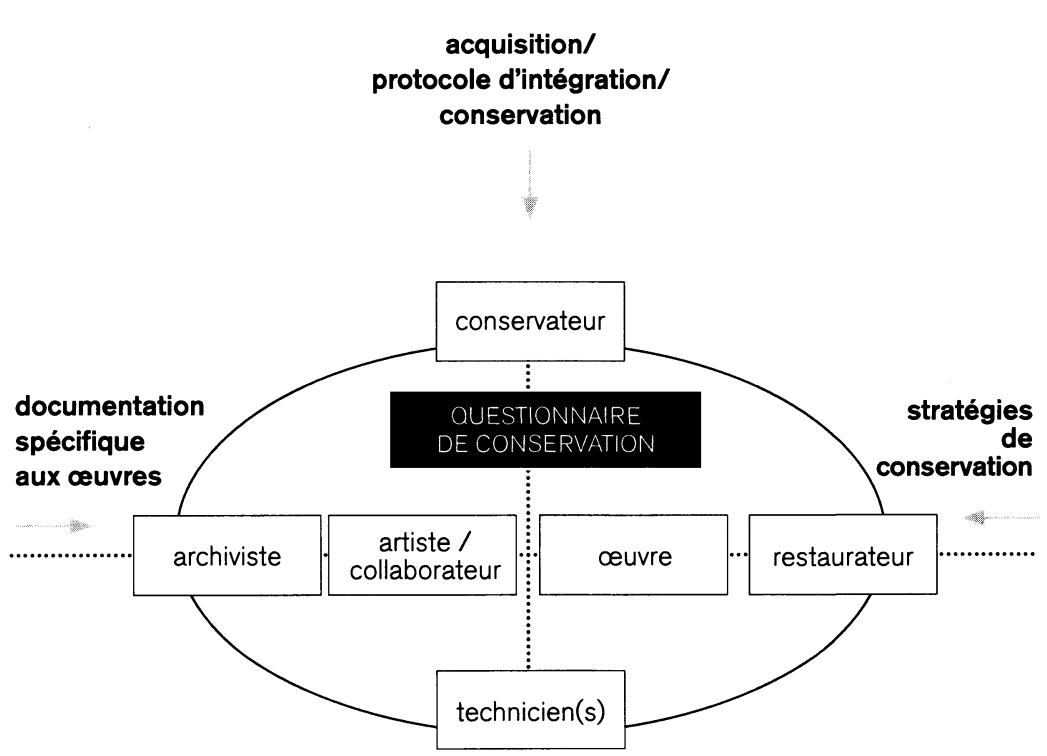

respect des exigences

d'installation 
Comme on a pu le constater au Musée d'art contemporain de Montréal et au Musée des beaux-arts du Canada, les approches avancées dans le contexte muséal dépendent de l'organisation du musée dans lequel l'œuvre est conservée. Les questions à poser sont multiples, d'autant plus que les recherches actuelles tendent clairement vers l'absence de protocole d'intégration et de mesures de conservation adaptés - problématique récurrente dans plusieurs institutions notamment en raison de la multiplicité des cas de figures apparus avec les nouvelles technologies -, plus particulièrement dans les petits et les moyens musées, et insistant, de ce fait, sur l'importance d'une documentation au cas par cas, documentation que l'on souhaiterait néanmoins commune, dans la mesure d'une certaine interopérabilité entre les institutions qui acquièrent ce type d'œuvres. Toutes ces mesures induisent une implication plus grande de la part du conservateur qui aura à prendre les décisions. Puisque le rôle de ce dernier est appelé à varier suivant la place accordée aux services de l'organisation dans laquelle il agit, il semblerait à propos de réévaluer l'articulation des charges de chacun en effectuant un retour sur les meilleures pratiques à observer, sans manquer de définir les facteurs et les stratégies d'intervention à considérer ${ }^{[37]}$ en fonction de leurs incidences sur la transmission et la mise en valeur de ce nouveau patrimoine culturel et en gardant bien évidemment à l'esprit l'intention de l'artiste et la manière dont il perçoit l'intégrité de son œuvre.
[37]

Ce dernier point pourrait s'avérer utile, comme en témoigne le Decision-making Model for the Conservation and Restoration of Modern Art (Foundation for the Conservation of Modern Art / Netherlands Institute for Cultural Heritage). Ce dispositif illustre distinctement les étapes à franchir ou les mesures d'accompagnement, allant, en introduction, de la prise d'information au sujet de l'intention de l'artiste (documentation et interview) à l'application d'un plan de conservation préventif et curatif comme mode final.

Voir à cet effet le diagramme "Decision-making Model for the Conservation and Restoration of Modern Art. Foundation for the Conservation of Modern Art » de l'INTERNATIONAL NETWORK FOR THE CONSERVATION OF CONTEMPORARY ART. The Decision-Making Model for the Conservation and Restoration of Modern and Contemporary Art. <http:// www.incca.org/dir003/incca/ $\mathrm{cmt} /$ text.nsf/0/3bd096d3c2 $48026 \mathrm{bc} 1256 \mathrm{af0004a6e8a}$ \$FILE/Decision-making\%20 Model.pdf>

(consulté en mars 2006). 


\section{Summary}

Conserving a Work with Technological Components

SERVICE DE TECHNOLOGIE DE LINFORMATION. BIBLIOTHĖQUE NATIONALE DU CANADA. La conservation de l'information numérique : ses divers aspects et la situation actuelle. $<$ http://cc.msncache.com/cache. $a p x ? q=1994680634543$ \&lang $=f r-$

CA\&FORM $=$ CVRE

(consulted in March 2006).

[39]

Monstration.

$<$ http://mitpress2.mit.edu/ejournals/LEA/MONOGRAPHS/

Monstration/monstration.htm $>$ (consulted in August 2006) [translated by Emma Healey]

"Preservation measures ensure that a document or artefact-digital or otherwise-is accessible in a usable form over time. ${ }^{\text {[ }}{ }^{381}$ This quote, taken from Alison Bullock's article on conserving information electronically, clearly illustrates the challenge that museum studies professionals face today. The rapid pace at which technology advances and its breakthroughs in various sectors of society demand that modifications be made regularly to various techniques and operating systems. With no let up on the sudden insurgence of different medias in the cultural community and the subsequent struggle that the concerned institutions must face-whether it be the new information technology and digital rights management of collections and exhibition centres or the digital knowledge in our libraries-, it is important to examine the difficulties of conserving works that have technological components and explore the strategies used to do so thus far.

How are we to regard these technological changes? Must we adapt the works of art created with technological elements at the rate of the evolution of their parts? What impact will this have on short- and long-term preservation and conservation? Given that these works and the multiple media from which they are made are by nature discontinuous and temporary, they pose serious problems for the institutions that are responsible for their conservation. Whether they are analogue or digital, mechanical or electrical, such works require us to explore possible ways to ensure their survival. That is the reason why we now see research projects being developed-sometimes isolated sometimes collective endeavours of grand proportions-whose main goal is to find solutions and make them readily available. Technology's rapid development leads to the degeneration of the material previously used to make the work. That premature degeneration starts a race against time, where there are various solutions and the ultimate decision-maker is often the artist.

In the era of possibilities, new strategies abound. We are no longer talking about conserving an "identical" " ${ }^{399}$ work of art, but rather about emulating, migrating, and reinterpreting it. Some solutions suggest finding equivalence by imitating the 
original appearance, upgrading the code, recreating the work. This has generated great debate concerning the initial aesthetics of the work and its intrinsic value and has raised questions about intellectual property. And while some are in favour of the possibility of migration, others are opposed it, as it tends too often to change the form of the work and create substantial reinterpretations and transmutations. In the quest for new materials, the use of the most recent technologies seems to have taken centre stage. And although those methods are radical, there is a push to use them. I will explore the positive and negative consequences of their application.

It has been widely recognized in museum practice that the multidisciplinary methods that artists have adopted in contemporary art and the range of materials and technologies that they use follow the lead of social actors, thus transforming and disrupting everyday conservation standards. Such transformations generate various questions that the museum must answer to avoid losing the first artistic expression or creation using technological components. The works that evolve from an outdated generation to another are transformed at such speed that the worst can be expected. They require more than just a global awareness; they necessitate a quick and efficient restructuring of present-day practices. Particularly, the major variations and metamorphoses in conservation practices that have taken place in recent years deserve to be studied and reviewed as quickly as they evolve. Without breaking with the past, we must see how a connection between the commonly used structures and the new questions brought up by conservation can be established.

Furthermore, the lack of integration protocol and conservation norms that are specifically adapted to such works demonstrates the importance of documenting on a case-by-case basis. Ideally, that information would be shared in order to maintain a certain interoperability between the documenting tools used by the museums that collect that type of works. Needless to say, such measures put the onus on the curator who will have to make certain decisions.

My analysis does not presume it offers ready-made solutions with regards to the practices that need to be put in place. It does, however, explore the possibility of creating a fundamental methodology, one based on the importance of corroborating with the artist concerning the conservation of his work and requesting him to complete a questionnaire to that effect. Much of my thought regarding the new concern with cultural heritage comes from this idea of the artist's involvement and it is supported by the questionnaire on different conservation practices that I created during my internship at the Musée d'art contemporain de Montréal (MACM), through meeting with specialized groups and thanks to the methodology of different revolutionary resources such as the Dictionnaire des arts médiatiques by the Universite du Québec à Montréal's Groupe de recherche en arts médiatiques (GRAM). 